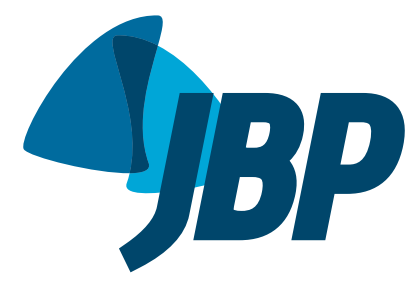

\title{
Clinical aspects in patients with pulmonary infection caused by mycobacteria of the Mycobacterium abscessus complex, in the Brazilian Amazon
}

\author{
José Tadeu Colares Monteiro ${ }^{1, a}$, Karla Valéria Batista Lima ${ }^{2, b}$, \\ Adriana Rodrigues Barretto ${ }^{3}$, Ismari Perini Furlaneto ${ }^{1,2, c}$, \\ Glenda Moraes Gonçalves ${ }^{3, \mathrm{~d}}$, Ana Roberta Fusco da Costa ${ }^{2, \mathrm{e}}$, \\ Maria Luiza Lopes ${ }^{2}$, Margareth Pretti Dalcolmo $4,5, \mathrm{f}$
}

1. Programa de Pós-Graduação em Biologia Parasitária na Amazônia Centro Universitário do Estado do Pará, Universidade do Estado do Pará, Belém (PA) Brasil.

2. Laboratório de Biologia Molecular Seção de Bacteriologia e Micologia, Instituto Evandro Chagas, Ananindeua (PA) Brasil.

3. Faculdade de Medicina, Universidade Federal do Pará, Belém (PA) Brasil.

4. Programa de Pós-Graduação, Pontifícia Universidade Católica do Rio de Janeiro, Rio de Janeiro (RJ) Brasil.

5. Centro de Referência Hélio Fraga, Escola Nacional de Saúde Pública Sérgio Arouca, Fundação Oswaldo Cruz, Rio de Janeiro (RJ) Brasil.

a. (D) http://orcid.org/0000-0002-7359-5178

b. (ID) http://orcid.org/0000-0001-5807-0392

c. (iD http://orcid.org/0000-0001-9941-0162

d. (D) http://orcid.org/0000-0002-0434-7664

e. (D) http://orcid.org/0000-0001-8719-4933

f. (iD) http://orcid.org/0000-0002-6820-1082

Submitted: 12 December 2016 Accepted: 16 October 2017.

Study carried out at the Ambulatório de Micobactérias Não Tuberculosas, Hospital Universitário João de Barros Barreto - HUJBB - Belém (PA) and in the Laboratório de Biologia Molecular, Seção de Bacteriologia e Micologia, Instituto Evandro Chagas, Ananindeua (PA) Brasil.

\begin{abstract}
Objective: To describe the clinical manifestations of patients with pulmonary infection caused by mycobacteria of the Mycobacterium abscessus complex (MABSC), and to compare these manifestations with those of patients infected with other nontuberculous mycobacteria (NTM). Methods: This was a retrospective cohort study involving 43 patients divided into two groups: the MABSC group, consisting of patients with pulmonary infection caused by MABSC ( $n=17$ ); and the NTM group, consisting of patients with pulmonary infection caused by NTM other than MABSC $(n=26)$. Patients were previously treated with a regimen of rifampin, isoniazid, pyrazinamide, and ethambutol before the diagnosis of NTM was confirmed by two culture-positive sputum samples. The nucleotide sequences of the $h s p 65,16 S$ rRNA, and/or rpoB genes were analyzed to identify the mycobacteria. Data were collected on demographic, clinical, and radiological characteristics, as well as on treatment responses and outcomes. Results: Loss of appetite was the only clinical manifestation that was significantly more common in the MABSC group than in the NTM group ( $p=0.0306$ ). The chance of having to use a second treatment regimen was almost 12 times higher in the MABSC group than in the NTM group. Treatment success was significantly higher in the NTM group than in the MABSC group (83.2\% vs. $17.6 \%$; $p$ 0.0001). The chance of recurrence was approximately 37 times higher in the MABSC group than in the NTM group. Conclusions: In the study sample, treatment response of pulmonary disease caused by MABSC was less favorable than that of pulmonary disease caused by other NTM.
\end{abstract}

Keywords: Nontuberculous mycobacteria/classification; Nontuberculous mycobacteria/ drug effects; Lung diseases.

\section{INTRODUCTION}

Nontuberculous mycobacteria (NTM) comprise a group of microorganisms that differ from Mycobacterium tuberculosis and $M$. leprae. There are currently more than 100 documented species of slowly or rapidly growing pathogenic or nonpathogenic NTM, many of which are environmental saprophytes and some of which are more related to opportunistic infections in immunocompromised individuals. ${ }^{(1)}$ The number of cases of NTM pulmonary infection has been increasing worldwide, and the most commonly involved pathogens are $M$. kansasii, M. abscessus, and the members of the M. avium complex (MAC). The M. abscessus complex
(MABSC) is characterized by rapidly growing mycobacteria in culture, represented by the subspecies $M$. abscessus subsp. abscessus, M. abscessus subsp. massiliense, and $M$. abscessus subsp. bolletii(2); the hallmark of this complex is the presence of genes conferring resistance to macrolides, the most widely described of these genes being the erm gene, except in the case of $M$. abscessus subsp. Massiliense, and this hinders treatment response in infected patients. ${ }^{(3)}$ In cases of MABSC pulmonary infection, protocols involve the use of macrolides (clarithromycin or azithromycin), aminoglycosides (amikacin), and there are regimens that include cefoxitin, imipenem, and tigecycline. ${ }^{(4)}$ Therefore, regimens can include up to two 
parenteral agents (amikacin, imipenem, tigecycline, or cefoxitin) for a long time (1 year). The profile of patients most affected by MABSC mycobacteria is not different from that of patients affected by other mycobacteria, the majority of whom are middle-aged women with structural lung changes (bronchiectasis)..$^{(5)}$ Regarding the epidemiology of NTM in Brazil, there are regional differences in the prevalence of the species involved. In a recent survey evaluating the incidence of cases of NTM among patients misdiagnosed with pulmonary tuberculosis, it was observed that $8 \%$ of those patients were infected with NTM in the Brazilian states of Bahia, Piauí, and Pará; in the Brazilian state of São Paulo, which accounted for most cases, $20 \%$ of the patients diagnosed with tuberculosis actually were infected with NTM. ${ }^{(6,7)}$ Since Brazil is a country of continental dimensions, demographic differences should be taken into account, and the lack of studies in this area in Brazil warrants further investigation for understanding the natural history of such patients in a regional context.

\section{METHODS}

This was a descriptive, analytical, retrospective cohort study using data on all patients diagnosed with NTM pulmonary infection who were followed between 2003 and 2013 at the NTM infection outpatient clinic of the Hospital Universitário João de Barros Barreto (HUJBB, João de Barros Barreto University Hospital), which is a referral center for the treatment of patients with multidrug-resistant tuberculosis and patients with NTM infection in the Brazilian state of Pará. The sample was selected by convenience. The study project was approved by the Research Ethics Committee of the HUJBB (CAAE: 44731115.6.3001.0017). Data were collected from patient charts and questionnaires that had been completed in monthly medical visits. Cases were reviewed starting at six months after treatment initiation, by using records from properly completed forms, including clinical evaluation data, bacteriological data (culture results), and radiological data (radiological records). The inclusion criteria were having a clinical, radiological, and biochemical diagnosis of NTM pulmonary infection - the last of the three being confirmed by two culture-positive sputum samples, in accordance with the American Thoracic Society (ATS) criteria-and having given written informed consent. We excluded cases in which there was concurrent isolation of $M$. tuberculosis. The identification of the mycobacterial species and subspecies involved, which was performed in the Molecular Biology Laboratory of the Evandro Chagas Institute, was based on the analysis of the nucleotide sequences of the hsp65, 16S rRNA, and/or $r p o B$ genes, as appropriate. The participants were divided into two groups: the MABSC group, consisting of 17 individuals with pulmonary infection caused by MABSC; and the NTM group, consisting of 26 individuals with pulmonary infection caused by NTM other than MABSC. The variables studied were categorized as demographic, clinical, and radiological (posteroanterior chest X-ray and/or HRCT of the chest).

Treatment responses were categorized after the use of the regimens available at the institution. The first regimen consisted of clarithromycin $(500 \mathrm{mg}$ p.o.; $12 / 12 \mathrm{~h})$, ethambutol (1,200 mg; $24 / 24 \mathrm{~h})$, and rifampin (600 mg p.o.; 24/24 h). The second regimen consisted of adding amikacin (500 mg i.m.; 3 times/ week) or streptomycin ( $1 \mathrm{~g}$ i.m.; 3 times/week) to the first regimen. The third regimen consisted of adding imipenem (500 mg i.v.; 6/6 h) to the second regimen. To assess outcomes, we took into account clinical improvement, as recorded on patient charts, and culture results, which were classified as culture conversion to negative, persistence of positivity (positive cultures for at least six months after treatment initiation), and positivity after initial conversion to negative (positive cultures for two consecutive months after initial conversion to negative). Radiological improvement as seen on chest X-ray or HRCT of the chest after treatment was the criterion defining cure until 2007 (prior to the 2007 ATS protocols). Sociodemographic and clinical characteristics were expressed as means and standard deviations, as medians and interquartile ranges, or as absolute and relative frequencies, as appropriate. The proportion of cases observed in each group, according to the NTM species isolated, was assessed by the chi-square test or G-test, whereas associations among categorical variables were examined by the chi-square test (or G-test), complemented by analysis of adjusted residuals, when necessary. The magnitude of association between outcome and condition was measured by the odds ratio. Differences among means were tested with the Student's t-test. All tests were performed with BioEstat 5.48, and results with $\mathrm{p} \leq 0.05$ were considered significant.

\section{RESULTS}

Of the 43 patients included in the study, 17 (39.5\%) belonged to the MABSC group and 26 (60.5\%) belonged to the NTM group. The mean age of the patients in the MABSC and NTM groups was $62.4 \pm 13.7$ years (range, 39-94 years) and $56.2 \pm 14.6$ years (range, 23-81 years), respectively, with the difference not being significant $(p=0.1715)$, and similar proportions of men $(23.5 \%$ vs. $34.6 \%)$ and women $(76.5 \%$ vs. $65.4 \%)$ were found in the two groups ( $p=0.6640)$.

With the exception of loss of appetite, which was significantly more common in the MABSC group than in the NTM group ( $p=0.0306)$, all other reported symptoms occurred in similar proportions in the groups $(p>0.05)$. The patients in the two groups had similar proportions of comorbidities at the time of diagnosis of the infection, and no significant associations were found between comorbidities and infection. There was a greater proportion of active smokers among the patients in the NTM group than among those in the MABSC group, and there was a greater proportion of 
passive smokers in the MABSC group than in the NTM group ( $\mathrm{p}<0.01$ for both; Table 1 ).

Table 2 shows the radiological findings at the time of diagnosis of NTM pulmonary disease, by study group. There were no statistically significant differences between the two groups regarding any of the study variables.

In the study sample, the chance of treatment nonadherence was 10 times higher in the MABSC group than in the NTM group (OR $=10.0 ; 95 \% \mathrm{CI}$ : 1.2-86.9). Regarding treatment response, the proportion of patients who did not respond to the first treatment regimen was significantly greater in the MABSC group than in the NTM group ( $p=0.0007)$, and, in the former group, 7 of the patients ( $41.2 \%$ ) had to use the second treatment regimen and $4(23.5 \%)$ had to use the third treatment regimen. In the NTM group, however, 20 of the 22 patients who completed the first treatment regimen $(83.2 \%)$ experienced cure, and only $2(9.0 \%)$ had to start the second treatment regimen (Table 3). Therefore, the chance of having to use a second treatment regimen was found to be almost 12 times higher in the MABSC group than in the NTM group (OR

Table 1. Patient distribution for comorbidities and lifestyle habits, by study group. Belém, Brazil, 2015 a $^{a}$

\begin{tabular}{|c|c|c|c|}
\hline \multirow[t]{2}{*}{ Variable } & \multicolumn{2}{|c|}{ Group } & \multirow[t]{2}{*}{ p* } \\
\hline & $\begin{array}{c}\text { MABSC } \\
(n=17)\end{array}$ & $\begin{array}{c}\text { NTM } \\
(n=26)\end{array}$ & \\
\hline \multicolumn{4}{|l|}{ Comorbidities } \\
\hline Absence & $3(17.7)$ & $6(23.1)$ & 0.3283 \\
\hline Diabetes & $1(5.9)$ & $0(0.0)$ & \\
\hline COPD and/or asthma and/or GERD & $2(11.7)$ & $4(15.4)$ & \\
\hline HIV infection & $0(0.0)$ & $4(15.4)$ & \\
\hline Isolated bronchiectasis & $6(35.3)$ & $7(26.9)$ & \\
\hline Associated bronchiectasis & $5(29.4)$ & $5(19.2)$ & \\
\hline \multicolumn{4}{|l|}{ Smoking } \\
\hline No & $4(23.5)$ & $6(23.1)$ & 0.0017 \\
\hline Active & $3(17.7)$ & $18(69.2)^{\dagger}$ & \\
\hline Passive & $8(47.1)^{\dagger}$ & $2(7.7)$ & \\
\hline Both & $2(11.7)$ & $0(0.0)$ & \\
\hline
\end{tabular}

MABSC: Mycobacterium abscessus complex; NTM: mycobacteria other than M. tuberculosis and MABSC; and GERD:

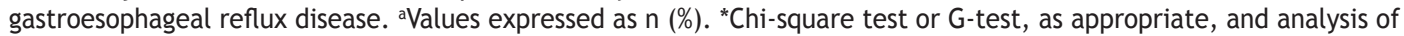
adjusted residuals, when necessary. ${ }^{\dagger}$ Cell with statistical significance.

Table 2. Pretreatment HRCT findings, by study group. Belém, Brazil, 2015. ${ }^{a}$

\begin{tabular}{|c|c|c|c|}
\hline \multirow[t]{2}{*}{ Variable } & \multicolumn{2}{|c|}{ Group } & \multirow[t]{2}{*}{ p* } \\
\hline & $\begin{array}{c}\text { MABSC } \\
(n=17)\end{array}$ & $\begin{array}{c}\text { NTM } \\
(n=26)\end{array}$ & \\
\hline \multicolumn{4}{|l|}{ Pulmonary involvement } \\
\hline Absent & $1(5.9)$ & $0(0.0)$ & \\
\hline Unilateral & $4(23.5)$ & $7(26.9)$ & 0.4772 \\
\hline Bilateral & $12(70.6)$ & $19(73.1)$ & \\
\hline \multicolumn{4}{|l|}{ Cavitation } \\
\hline Absent & $8(47.1)$ & $11(42.3)$ & 0.7369 \\
\hline Single & $4(23.5)$ & $9(34.6)$ & \\
\hline Multiple & $5(29.4)$ & $6(23.1)$ & \\
\hline \multicolumn{4}{|l|}{ Distribution of cavitation } \\
\hline Unilobar & $7(77.8)$ & $10(66.7)$ & 0.2555 \\
\hline Bilobar & $0(0.0)$ & $3(20.0)$ & \\
\hline Multilobar & $2(22.2)$ & $2(13.3)$ & \\
\hline \multicolumn{4}{|l|}{ Bronchiectasis } \\
\hline Absent & $1(5.9)$ & $4(15.4)$ & 0.3376 \\
\hline Present & $11(64.7)$ & $11(42.3)$ & \\
\hline Present (subsequent NTM infection) & $5(29.4)$ & $11(42.3)$ & \\
\hline \multicolumn{4}{|l|}{ Radiological presentation } \\
\hline Fibrocavitary & $7(41.2)$ & $14(53.8)$ & 0.6163 \\
\hline Nodular bronchiectasis & $10(58.8)$ & $12(46.2)$ & \\
\hline
\end{tabular}

MABSC: Mycobacterium abscessus complex; and NTM: mycobacteria other than M. tuberculosis and MABSC. a Values expressed as $\mathrm{n}(\%)$. ${ }^{*}$ Chi-square test or $\mathrm{G}$-test, as appropriate. 
Table 3. Treatment regimens used and patient outcomes observed, by study group. Belém, Brazil, 2015.

\begin{tabular}{|c|c|c|c|c|}
\hline \multirow[t]{2}{*}{ Variable } & \multicolumn{2}{|c|}{ Group $^{a}$} & \multirow[t]{2}{*}{ p* } & \multirow[t]{2}{*}{ OR (95\% CI) } \\
\hline & $\begin{array}{c}\text { MABSC } \\
(n=17)\end{array}$ & $\begin{array}{c}\text { NTM } \\
(n=26)\end{array}$ & & \\
\hline \multicolumn{5}{|l|}{ Regimen used ${ }^{b}$} \\
\hline 1st regimen & $6(35.3)$ & $20(91.0)^{\ddagger}$ & & 1.0 \\
\hline 2 nd regimen & $7(41.2)^{\dagger}$ & $2(9.0)$ & 0.0007 & $11.70(1.9-71.8)$ \\
\hline 3rd regimen & $4(23.5)^{\dagger}$ & $0(0.0)$ & & - \\
\hline \multicolumn{5}{|l|}{ Primary outcome ${ }^{c}$ (after 1 st regimen) } \\
\hline Clinical improvement and cure & $3(17.6)$ & $20(83.2)^{\dagger}$ & $<0.0001$ & 1.00 \\
\hline Dropout & $3(17.6)$ & $2(8.4)$ & & $10.00(1.2-86.9)$ \\
\hline Treatment failure & $11(64.8)^{\dagger}$ & $2(8.4)$ & & $36.70(5.3-253.8)$ \\
\hline \multicolumn{5}{|l|}{ Secondary outcome } \\
\hline Clinical improvement and cure & $10(91.0)$ & $2(100)$ & 0.3917 & 1.0 \\
\hline Death & $1(9.0)$ & $0(0.0)$ & & - \\
\hline
\end{tabular}

MABSC: Mycobacterium abscessus complex; NTM: mycobacteria other than $M$. tuberculosis mycobacteria and MABSC; 1st regimen: rifampin, ethambutol, and clarithromycin; 2nd regimen: 1st regimen plus amikacin or

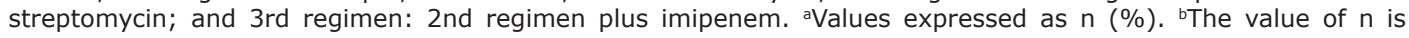
different in the NTM group because of two deaths and two dropouts for which there was no information on primary outcome. 'The value of $\mathrm{n}$ is different in the NTM group because of two deaths of unknown cause. *G-test and analysis of adjusted residuals, when necessary. ${ }^{+}$Cell with statistical significance.

$=11.70 ; 95 \% \mathrm{CI}: 1.9-71.8)$. We emphasize that none of the patients in the NTM group had to use the third treatment regimen (second treatment regimen plus imipenem). Regarding primary outcomes, there was a significantly higher frequency of recurrence in the MABSC group than in the NTM group ( $p<0.0001$ ), and the chance of recurrence was approximately 37 times higher in the MABSC group than in the NTM group ( $O R=36,7 ; 95 \% \mathrm{CI}: 5.3-253.8)$. In contrast, an evaluation of cases of clinical improvement and cure after completion of the first treatment regimen showed that the proportion of these cases was significantly greater in the NTM group than in the MABSC group $(p<0.0001)$. These data suggest that pulmonary disease caused by MABSC is more aggressive and has a worse prognosis than pulmonary disease caused by other NTM (Table 3). We emphasize that there were 2 deaths of unknown cause (not related to NTM infection) in the NTM group, and, therefore, these patients were excluded from the comparisons. Regarding secondary outcomes-obtained after the use of the second regimen or after administration of the third regimen-91.0\% $(10 / 11)$ of the patients in the MABSC experienced clinical improvement and cure, and 9.0\% (1/11) died; the patient who died was among the patients who used imipenem (third regimen). One of the patients discharged after the use of the third regimen required surgical treatment. Of the patients in the NTM group who received the second treatment regimen $(n=2)$, all $(100 \%)$ achieved treatment success. However, there were no statistically significant differences regarding these outcomes between the groups evaluated ( $p=$ 0.3917).

\section{DISCUSSION}

In view of the increasing number of cases of NTM infection, several studies have been conducted to analyze the condition and its epidemiology. ${ }^{(8-10)}$ Of the
43 patients included in the present study, 39.5\% had MABSC infection and $60.5 \%$ had pulmonary disease caused by other NTM, the most prevalent of which were MAC mycobacteria. The prevalence of pulmonary disease caused by MAC and by MABSC found in our study is in line with that reported in recent surveys conducted in the United States, where MABSC infection is second only to MAC infection, accounting for 2.6$13.0 \%$ of all cases of NTM pulmonary infection, with an annual prevalence of $<1 / 100,000$ population. ${ }^{(4)}$ Regarding pulmonary disease caused by MABSC subspecies, our results were similar to those reporting that the proportion of cases of $M$. abscessus subsp. abscessus would be similar to that of cases of $M$. abscessus subsp. massiliense, whereas M. abscessus subsp. bolletii is rarely identified. $(4,11,12)$ Conditions predisposing to disease include previous treatment for pulmonary tuberculosis and structural pulmonary changes, represented here by bronchiectasis. ${ }^{(9,13,14)}$ In a study comparing clinical variables between patients with pulmonary disease caused by M. abscessus subsp. massiliense and those with pulmonary disease caused by $M$. abscessus subsp. abscessus, no significant differences were found, ${ }^{(15)}$ which is in contrast with the findings of the present study, in which loss of appetite was more common among those infected with MABSC mycobacteria. Regarding multiple treatments with the rifampin-isoniazid-pyrazinamide-ethambutol (RHZE) regimen for tuberculosis in patients infected with NTM, this is due to the fact that identification of mycobacteria in cultures is not mandatory in Brazil. Therefore, patients who received previous treatments for pulmonary tuberculosis experienced a higher frequency of asthenia, and this frequency was significantly (3-4 times) higher in those improperly treated with the RHZE regimen $(p=0.0491)$. In the Amazon, the great distances and the few centers equipped for the follow-up of patients infected with NTM are factors 
that result in a delay in appropriate diagnosis and treatment of these patients. ${ }^{(16)}$

Although our study found no association between bronchiectasis and the study groups, bronchiectasis was the most prevalent comorbidity in both groups, proving the strong association between NTM infection and bronchiectasis. In a retrospective observational study that included 107 patients with pulmonary disease caused by MABSC, bronchiectasis and cavitation were found in $98 \%$ and $44 \%$ of the patients, respectively. (17) In that study, the nodular bronchiectasis form was associated with patients without previous treatment for pulmonary tuberculosis ( $p=0.0325$ ), which may be due to the fact that physicians associate cavitation with pulmonary tuberculosis and therefore request sputum smear microscopy for AFB early, whereas the presence of nodules and/or bronchiectasis requires a more thorough etiological investigation, demanding specialized evaluation. ${ }^{(18)}$ Regarding the treatment of MABSC pulmonary infections, high-level resistance to the drugs in the RHZE regimen has been extensively described. In addition, resistance mechanisms similar to those of macrolide and aminoglycoside resistance are of note, impairing treatment response in this group of patients and worsening their prognosis. ${ }^{(19)}$ The results of the present analysis show that the proportion of cases that did not respond to the first treatment regimen and required a second or third regimen was almost 12 times higher in the MABSC group than in the NTM group. Chief among the factors complicating this patient follow-up phase is the lack of susceptibility testing, which, because of technical and institutional limitations, was not performed; the result was that most regimens were initiated on the basis of few existing clinical protocols or of consultation with experienced specialists, as recommended in the ATS document itself, and therefore relying on a low level of evidence. ${ }^{(20)}$ We observed that, in the NTM group, none of the patients had to use the third regimen containing imipenem, which may be explained by the fact that MAC has a different susceptibility pattern, showing susceptibility to regimens containing macrolides and antituberculosis drugs, which is well established, including in the ATS consensus statement. ${ }^{(20)}$ New publications, subsequent to those of the ATS, recommend the use of intermittent therapy administered thrice weekly in an attempt to increase patient adherence, ${ }^{(10)}$ because of the long treatment time and the possible side effects of the drugs; in our study sample, the chance of treatment nonadherence was 10 times higher in the MABSC group than in the NTM group. The patients in the NTM group, which was comprised mostly of individuals infected with MAC, achieved treatment success after the use of the second regimen, confirming literature findings that prognosis is better in cases of pulmonary disease caused by MAC or other NTM than in those of pulmonary disease caused by MABSC. ${ }^{(21)}$ In cases of infection with $M$. massiliense-which, according to the literature, appear to exhibit a susceptibility pattern when treated with regimens containing macrolides-the chance of having to use a second treatment regimen is 10 times higher than it is in cases of infection with other NTM (95\% CI: 1.4-74.5). ${ }^{(21)}$

MAC organisms are the most relevant slowly growing mycobacteria, and, according to recently published data, individuals infected with these mycobacteria have a better prognosis and a survival of 13 years. ${ }^{(20,21)}$ In our study sample, the cure rate achieved with the first treatment regimen (clarithromycin, ethambutol, and rifampin) was high (83.2\%) among the patients in the NTM group (which was mostly comprised of individuals infected with MAC), of whom only $2(9.0 \%)$ had to start the second regimen, and the difference between the two groups was significant. The results of the present study, despite the technical limitations due to the lack of susceptibility testing, as well as the limitations due to the study's retrospective design and the fact that is was conducted in a referral hospital, which may have introduced selection and information bias, are in line with those of most published studies on the course of illness in patients with pulmonary disease caused by MABSC. (22-24) The unfavorable outcomes of long-term treatments with several prescribed regimens, together with important adverse effects, demonstrate the urgent need for a laboratory network that can identify NTM species and perform early susceptibility testing, so as to enable that specific standardized protocols be evaluated and instituted.

\section{REFERENCES}

1. Nunes-Costa D, Talarico S, Dalcolmo $M$, Correia-Neves $M$, Empadinhas N. The looming tide of nontuberculous mycobacterial infection in Portugal and Brazil. Tuberculosis (Edinb). 2016;96:107-19. https://doi.org/10.1016/..tube.2015.09.006

2. Nie W, Duan H, Huang H, Lu Y, Bi D, Chu N. Species identification of Mycobacterium abscessus subsp. abscessus and Mycobacterium abscessus subsp. bolletii using rpoB and hsp65, and susceptibility testing to eight antibiotics. Int J Infect Dis. 2014;25:170-4. https://doi. org/10.1016/j.jijid.2014.02.014

3. Rubio M, March F, Garrigó M, Moreno C, Español M, Coll P. Inducible and Acquired Clarithromycin Resistance in the Mycobacterium abscessus Complex. PloS One. 2015;10(10):e0140166. https://doi. org/10.1371/journal.pone.0140166

4. Lee MR, Sheng WH, Hung CC, Yu CJ, Lee LN, Hsueh PR Mycobacterium abscessus Complex Infections in Humans. Emerg Infect Dis. 2015;21(9):1638-46. https://doi.org/10.3201/2109.141634
5. Cândido PH, Nunes Lde S, Marques EA, Folescu TW, Coelho FS, de Moura VC, et al. Multidrug-resistant nontuberculous mycobacteria isolated from cystic fibrosis patients. J Clin Microbiol. 2014;58(8):2990-7. https://doi.org/10.1128/JCM.00549-14

6. Williams MM, Yakrus MA, Arduino MJ, Cooksey RC, Crane CB, Banerjee $S$, et al. Structural analysis of biofilm formation by rapidly and slowly growing nontuberculous mycobacteria. Appl Environ Microbiol. 2009;75(7):2091-8. https://doi.org/10.1128/AEM.00166-09

7. de Mello KG, Mello FC, Borga L, Rolla V, Duarte RS, Sampaio EP et al. Clinical and therapeutic features of pulmonary nontuberculous mycobacterial disease, Brazil, 1993-2011. Emerg Infect Dis. 2013;19(3):393-9.

8. Ayres M, Ayres Junior M, Ayres DL, Santos AS. Bioestat 5.0 Aplicações estatísticas nas áreas das ciências biomédicas. Belém: ONG Mamiraua; 2007.

9. Sousa S, Bandeira M, Carvalho PA, Duarte A, Jordao L. 
Nontuberculous mycobacteria pathogenesis and biofilm assembly Int J Mycobacteriol. 2015;4(1):36-43. https://doi.org/10.1016/j. ijmyco.2014.11.065

10. Mcshane PJ, Glassroth J. Pulmonary Disease Due To Nontuberculous Mycobacteria: Current State and New Insights. Chest. 2015;148(6):1517-1527. https://doi.org/10.1378/chest.15-0458

11. Adékambi T, Drancourt M. Mycobacterium bolletii respiratory infections. Emerg Infect Dis. 2009;15(2):302-5. https://doi org/10.3201/eid1502.080837

12. Jeong BH, Kim SY, Jeon $K$, Huh HJ, Ki CS, Lee NY, et al. The First Korean Case of Nontuberculous Mycobacterial Lung Disease Caused by Mycobacterium abscessus Subspecies bolletii in a Patient with Bronchiectasis. Tuberc Respir Dis (Seoul). 2014;76(1):30-3. https:// doi.org/10.4046/trd.2014.76.1.30

13. Prevots DR, Marras TK. Epidemiology of human pulmonary infection with nontuberculous mycobacteria: a review. Clin Chest Med. 2015;36(1):13-34. https://doi.org/10.1016/..ccm.2014.10.002

14. Thomson R, Tolson C, Carter R, Coulter C, Huygens F, Hargreaves M. Isolation of nontuberculous mycobacteria (NTM) from household water and shower aerosols in patients with pulmonary disease caused by NTM. J Clin Microbiol. 2013;51(9):3006-11. https://doi. org/10.1128/JCM.00899-13

15. Harada T, Akiyama Y, Kurashima A, Nagai H, Tsuyuguchi K, Fujii T, et al. Clinical and microbiological differences between Mycobacterium abscessus and Mycobacterium massiliense lung diseases. J Clin Microbiol. 2012;50(11):3556-61. https://doi.org/10.1128/JCM.01175-12

16. Jarrand J, Levin A, Zhang L, Huitt G, Mitchell JD, Daley CL. Clinical and microbiologic outcomes in patients receiving treatment for Mycobacterium abscessus pulmonary disease. Clin Infect Dis. 2011:52(5):565-71. https://doi.org/10.1093/cid/ciq237

17. Chung MJ, Lee KS, Koh WJ, Lee JH, Kim TS, Kwon OJ, et al. Thinsection CT findings of nontuberculous mycobacterial pulmonary diseases: comparison between Mycobacterium avium-intracellulare complex and Mycobacterium abscessus infection. J Korean Med Sci. 2005;20(5):777-83. https://doi.org/10.3346/jkms.2005.20.5.777

18. Chu H, Li B, Zhao L, Huang D, Xu J, Zhang J, et al. Tree-in-bud pattern of chest $\mathrm{CT}$ images for diagnosis of Mycobacterium abscessus. Int $J$ Clin Exp Med. 2015; 8(10):18705-12. eCollection 2015.

19. Philley JV, Griffith DE. Treatment of slowly growing mycobacteria Clin Chest Med. 2015;36(1):79-90. https://doi.org/10.1016/j. ccm.2014.10.005

20. Maurer FP, Castelberg C, Quiblier C, Böttger EC, Somosköv A. Erm(41)-dependent inducible resistance to azithromycin and clarithromycin in clinical isolates of Mycobacterium abscessus. $J$ Antimicrob Chemother. 2014;69(6):1559-63. https://doi.org/10.1093/ jac/dku007

21. Shin SJ, Choi GE, Cho SN, Woo SY, Jeong BH, Jeon K, et al. Mycobacterial genotypes are associated with clinical manifestation and progression of lung disease caused by Mycobacterium abscessus and Mycobacterium massiliense. Clin Infect Dis. 2013;57(1):32-9. https://doi.org/10.1093/cid/cit172

22. Olivier KN, Shaw PA, Glaser TS, Bhattacharyya D, Fleshner $M$, Brewer CC, et al. Inhaled amikacin for treatment of refractory pulmonary nontuberculous mycobacterial disease. Ann Am Thorac Soc. 2014;11(1):30-5. https://doi.org/10.1513/AnnalsATS.201307$2310 \mathrm{C}$

23. Plotinsky RN, Talbot E, von Reyn CF. Proposed definitions for epidemiologic and clinical studies of Mycobacterium avium complex pulmonary disease. PloS One. 2013;8(11):e77385. https://doi. org/10.1371/journal.pone.0077385

24. Kotilainen $H$, Valtonen $V$, Tukiainen $P$, Poussa $T$, Eskola J, Järvinen A. Clinical findings in relation to mortality in non-tuberculous mycobacterial infections: patients with Mycobacterium avium complex have better survival than patients with other mycobacteria Eur J Clin Microbiol Infect Dis. 2015;34(9):1909-18. https://doi. org/10.1007/s10096-015-2432-8 